\title{
Variability and Association of Capsaicin and Oleoresin on Seed Quality in Hot Pepper (Capsicum spp)
}

\author{
K. S. Nagaraju ${ }^{1 *}$, K. P. R. Prasanna ${ }^{1}$, A. Mohan Rao ${ }^{2}$ and K. Aruna ${ }^{2}$ \\ ${ }^{1}$ Department of Seed Science and Technology, ${ }^{2}$ Department Genetics and Plant Breeding, \\ University of Agricultural Sciences, Bangalore, India \\ *Corresponding author
}

\section{A B S T R A C T}

\section{Keywords \\ Hot pepper, \\ Variability, \\ Heritability, \\ Association, Path \\ Coefficient and \\ Seed germination, \\ Fruit rot incidence \\ Article Info \\ Accepted: \\ 04 October 2020 \\ Available Online: \\ 10 November 2020}

\begin{abstract}
Seventeen hot pepper characters studied manifested higher level of GCV except for seed germination (16.47\%) and seedling dry weight (13.12\%) manifested moderate level of GCV. Phenotypic Co-efficient of Variation (PCV) for germination (16.79\%) and seedling dry weight (13.12\%) manifested moderate level of PCV. Significant positive correlation was recorded with germination while electrical conductivity $(-0.8125)$, fresh ungerminated seeds $(-0.8890)$, capsaicin (-0.7482) showed significant negative correlation with seed germination. Path coefficient analysis revealed that, at phenotypic level, seedling length (0.7297) recorded the highest positive direct effect on vigour index I, followed by germination per cent $(0.3452)$ which were of considerable magnitude. Number of seed fruit $^{-1}(-0.1266)$, electrical conductivity (-0.0627), total dehydrogenase activity (-0.0312), vigour index II (-0.0339) and ASTA color value (-0.0872) recorded the negative direct effect on fruit rot disease incidence on ripe fruits.
\end{abstract}

\section{Introduction}

Hot pepper (Capsicum annuum L.) is highly valued as a vegetable and spice, as well as commercial crop and becoming increasingly important in terms of production and consumption. The Crop can be raised all round the year with the benefits of faster, cleaner production and a premium quality end product. At the same time, chilies are rich source of vitamin ' $A$ ' and ' $C$ '. Many chilli pepper constituents have importance for nutritional value, flavour, aroma, texture, and colour. Besides, Fruits of chilli comprises numerous chemicals components including steam-volatile oil, fatty oils, capsaicinoids, carotenoids, vitamins, protein, fiber, and mineral elements (Bosland and Votava, 2000).

Two important quality attributes of hot pepper is pungency imparted by the presence of capsaicinoids and the color due to carotenoids. Capsaicin is a unique alkaloid of the plant kingdom restricted to the genus Capsicum. Capsaicin is the pungency factor, a 
bioactive molecule of food and of medicinal importance. Capsaicin is used as a counterirritant, anti-arthritic, analgesic, antioxidant, and anticancer agent. The rich supplies of chilli peppers carotenoids contribute to food nutritional value and color (Anon. 2003).

Pungency or "heat" found in Capsicum fruit results from the biosynthesis and accumulation of alkaloid compounds known as capsaicinoids in the dissepiments, placental tissue adjacent to the seeds. Pepper cultivars vary with respect to their level of pungency because of quantitative and qualitative variation in capsaicinoid content. A total of six different main effect QTL affecting capsaicinoid content were identified that are mapped to chromosomes 3, 4 and 7 (Arnon, 2006).

The hot flavor of chilies is due to presence of a group of seven closely related compounds called capsaicinoids, but capsaicin (8-methyl$\mathrm{N}$-vanillyl-6-nonenamide) and dihydrocapsaicin are responsible for approximately $90(\%)$ of the pungency $1-3$. Chilli hotness is measured in Scoville Heat Units (SHU) which is originally a subjective measure but today, chilli hotness is more frequently determined by HPLC (high performance liquid chromatography), whose results can be correlated to traditional Scoville ratings: the conversion generally accepted is that 15 Scoville units is equal to 1 ppm capsaicin plus capsaicinoids (Ritesh et al., 2000).

Tewksbury and Nabhan (2001) investigated directed deterrence by capsaicin in chilies (Capsicum) and revealed that capsaicin, the chemical responsible for the fruit's peppery heat, selectively discourages vertebrate predators without deterring more effective seed dispersers. And opined that, worldwide popularity of chilies has prompted numerous investigations of the biological properties of capsaicin yet its evolutionary significance in the plants that produce it has not been examined completely.

Tewksbury et al., (2008) showed that chemical defense of chilli ripe fruit reflects variation in the risk of microbial attack in Capsicum chacoense. In population producing varied contents of capsaicinoids in naturally polymorphic proportion, showed that variation is directly linked to variation in the damage caused by Fusarium fungal pathogen on chilli seeds and experimentally demonstrated that capsaicinoids protect chilli seeds from Fusarium. They also opined that pungency in chillies may be an adaptive response to selection by a microbial pathogen.

\section{Materials and Methods}

Forty seven (47) hot pepper genotypes belonging to three Capsicum species viz., Capsicum annuum var. longum, Capsicum frutescens and Capsicum chinense were grown during March, 2008 using Completely Randomized Design following recommended package of practices (Anon. 2006). These genotypes showing consistent morphological characters confirming the true to type were subjected to self-pollination and the resulted seeds were used in the present studies.

The following observations were record. viz., No. of seed fruit ${ }^{-1}, 1000$ seed weight $(\mathrm{g})$, Electrical conductivity $\left(\mu \mathrm{S} \mathrm{cm}^{-1} \mathrm{~g}^{-1)}\right.$, Total dehydrogenase activity (TDH), Germination $(\%)$, Fresh ungerminated seeds (\%), Seedling length $(\mathrm{cm})$, Seedling vigour index-I, Seedling dry weight $(\mathrm{mg})$. Seedling vigour index II, Seed infection (\%), Fruit rot disease incidence on ripe fruits, Capsaicin $(\mathrm{mg} / \mathrm{g})$ (Sadasivam and Manickam, 1996 by calorimetric method), $\alpha$-amylase activity ( $\mu \mathrm{g}$ $/ \mathrm{ml} / \mathrm{min}$.) as outlined by Sadasivam and Manickam, 1996, Total soluble seed protein 
content as per Lowry et al., 1951, Oleoresin (\%) as envisaged by Ranganna, 1996 with necessary modifications and ASTA color value.

Analysis of variance (ANOVA) was carried out as per the method suggested by Cochran and Cox (1959). The phenotypic and genotypic coefficient of variation was computed as per Burton and Dewane (1953) for low moisture stress. Heritability estimate was calculated using the formula (Hanson et al., 1956) as per cent mean. Heritability percentage was categorized as given by Robinson et al., 1949. Genetic advance was calculated by using formula given by Johnson et al., (1955) and was expressed as per cent of mean. To estimate the degree of association between the traits studied, phenotypic correlation was computed by using the formula given by Sundararaj et al., (1972). Path coefficient analysis was carried out as suggested by Wright (1921) and illustrated by Dewey and $\mathrm{Lu}$ (1959) in $\mathrm{F}_{2}$ generations. Standard path coefficients, which are standardized partial regression coefficients, were obtained by solving the following sets of ' $\mathrm{P}$ ' simultaneous equation by "DOOLITTLE TECHNIQUE" as described by Goulden, 1959. The data obtained from the experiment were statistically analyzed by using appropriate ANOVA by WINDOWSTAT version 9.1 from INDOSTAT services, Hyderabad licensed to Agriculture Knowledge Management Unit, GKVK, UAS, Bangalore.

\section{Results and Discussion}

\section{Genetic variability and seed quality parameters}

Phenotypic coefficient of variation (PCV) and genotypic coefficient of variation (GCV)

Number of seed fruit $^{-1}, 1000$ seed weight, electrical conductivity, total dehydrogenase activity, fresh ungerminated seeds, Seedling lengh, seedling vigour index I, seedling vigour index II, seed infection, fruit rot disease incidence on ripe fruits, $\alpha$-amylase activity, total soluble seed protein content, capsaicin, oleoresin, ASTA colour value manifested higher level of PCV and GCV while germination per cent and seedling dry weight manifested moderate level of PCV and GCV with close correspondence between them indicated lesser influence of environment on these traits thus selection for these traits to study seed quality attributes at in hot peppers cultivars will be effective (Table 1).

Present results are in accordance with the earlier reports of high PCV and GCV for no. of seed fruit ${ }^{-1}$ (Mini et al., 2004; Yudhvir and Madhu Sharma, 2008), oleoresin (Pitchumuthu and Pappiah,1995), fresh unfermented seeds (Claudinei et al., 2006), fruit rot disease incidence on ripe fruits (Jayashree et al., 2007 and Gadal et al., 2003), total soluble seed protein content (Yudhvir et al., 2009), capsaicin (Hedau et al., 2008), oleoresin and ASTA colour value (Mini et al., 2004; Naresh et al., 2013; Shiva et al., 2014).

\section{Heritability and genetic advance}

Electrical conductivity, total dehydrogenase activity, seedling dry weight, seed infection, fruit rot disease incidence on ripe fruits, total soluble seed protein content, capsaicin, ASTA color value, number of seed/fruit, 1000 seed weight, germination per cent, fresh ungerminated seeds, seedling length, seedling vigour index I, seedling vigour index II, $\alpha$ amylase activity and oleoresin manifested higher heritability and GAM indicated the role of additive gene effects and hence selection would be rewarding for improvement of such traits (Table 1). 
Whereas, the present study reported presence of moderate PCV and GCV for germination, seedlings dry weight, $\alpha$-amylase activity, seed infection, seedling length, seedling vigour index I and II in the studied hot pepper genotypes.

Similarly, many researchers observed such presence of high GCV, PCV and high heritability coupled with genetic advance in
(\%) grand mean viz., Sarkar et al., (2009) for number of seeds fruit ${ }^{-1}$; Manju and Srilathakumari (2002) for seed yield per fruit; Mini and Khader (2004) for 100 seed weight; Verma et al., (2004) for days to 50 (\%) germination; Yudhvir Singh and Madhu Sharma (2008) for oleoresin and capsaicin content in hot peppers.

Table.1 Genetic variability parameters in hot pepper

\begin{tabular}{|c|c|c|c|c|c|c|c|c|}
\hline \multirow[t]{2}{*}{ Sl. No. } & \multirow[t]{2}{*}{ Characters } & \multicolumn{2}{|c|}{ Range } & \multirow{2}{*}{$\begin{array}{l}\text { Grand } \\
\text { Mean }\end{array}$} & \multirow{2}{*}{$\begin{array}{c}\text { GCV } \\
(\%)\end{array}$} & \multirow{2}{*}{$\begin{array}{l}\text { PCV } \\
(\%)\end{array}$} & \multirow{2}{*}{$\begin{array}{c}\mathbf{h}^{2}(\%) \\
(\text { Broad Sense) }\end{array}$} & \multirow[t]{2}{*}{ GAM } \\
\hline & & Highest & Lowest & & & & & \\
\hline 1 & No. of seed fruit ${ }^{-1}$ & 242.33 & 17.67 & 91.41 & 48.99 & 50.32 & 95 & 98.26 \\
\hline 2 & 1000 seed weight $(\mathrm{g})$ & 9.74 & 0.3 & 5.49 & 27.65 & 28.49 & 94 & 55.28 \\
\hline 3 & Electrical conductivity $\left(\mu \mathrm{S} \mathrm{cm}^{-1} \mathrm{~g}^{-1)}\right.$ & 13.48 & 0.33 & 2.15 & 144.13 & 144.14 & 100 & 296.91 \\
\hline 4 & Total dehydrogenase activity (TDH) & 3.18 & 0.88 & 2.17 & 28.44 & 28.44 & 100 & 58.57 \\
\hline 5 & Germination (\%) & 96.33 & 38.67 & 77.2 & 16.47 & 16.79 & 96 & 33.27 \\
\hline 6 & Fresh ungerminated seeds (\%) & 9.73 & 0.71 & 1.83 & 136.81 & 137.23 & 99 & 280.98 \\
\hline 7 & Seedling length $(\mathrm{cm})$ & 17.88 & 5.87 & 12.81 & 21.44 & 22.4 & 92 & 42.28 \\
\hline 8 & Seedling vigour index-I & 1488.67 & 283.33 & 1015.63 & 30.49 & 31.31 & 95 & 61.18 \\
\hline 9 & Seedling dry weight (mg) & 14.69 & 8.11 & 11.48 & 13.12 & 13.12 & 100 & 27.03 \\
\hline 10 & Seedling vigour index II & 1235.67 & 344.67 & 901.43 & 24.41 & 25.17 & 94 & 48.78 \\
\hline 11 & Seed infection $(\%)$ & 82 & 0 & 31.57 & 68.06 & 68.18 & 100 & 139.97 \\
\hline 12 & Fruit rot disease incidence on ripe fruits & 94.53 & 0 & 41.3 & 70.84 & 70.97 & 100 & 145.66 \\
\hline 13 & $\alpha$-amylase activity $\left(\mathrm{mg}^{-1} \mathrm{ml}^{-1}-\mathrm{min}^{-1}.\right)$ & 37.03 & 12 & 26.68 & 24.42 & 24.63 & 98 & 49.85 \\
\hline 14 & Total soluble seed protein content & 18.05 & 6.38 & 11.65 & 29.24 & 29.24 & 100 & 60.24 \\
\hline 15 & Capsaicin $\left(\mu \mathrm{g} \mathrm{g}^{-1}\right)$ & 417 & 27 & 178.95 & 54.43 & 54.55 & 100 & 111.85 \\
\hline 16 & Oleoresin $(\%)$ & 22.58 & 3.2 & 11.65 & 35.82 & 35.99 & 99 & 73.43 \\
\hline 17 & ASTA color value & 165.1 & 2.43 & 68.96 & 69.77 & 69.77 & 100 & 143.7 \\
\hline $\begin{array}{l}\text { Where, } \\
\mathbf{X}_{1} \\
\mathbf{X}_{2} \\
\mathbf{X}_{3} \\
\mathbf{X}_{4} \\
\mathbf{X}_{5} \\
\mathbf{X}_{6} \\
\mathbf{X}_{7} \\
\mathbf{X}_{8} \\
\mathbf{X}_{9} \\
\mathbf{X}_{10}\end{array}$ & $\begin{array}{l}\text { : Number of seed fruit } \\
: \text { Test weight }(\mathrm{g}) \\
\text { : Electrical conductivity }\left(\mathrm{mS} \mathrm{cm}^{-1} \mathrm{~g}^{-1}\right) \\
\text { : Total dehydrogenase activity }\left(\mathrm{A}^{0} 480 \mathrm{~nm}\right) \\
: \text { Germination }(\%) \\
: \text { Fresh ungerminated seeds }(\%) \\
: \text { Seedling length }(\mathrm{cm}) \\
\text { : Seedling vigour index-I } \\
: \text { Seedling dry weight }(\mathrm{mg}) \\
\text { : Seedling vigour index-II }\end{array}$ & & $\begin{array}{l}\mathbf{X}_{11} \\
\mathbf{X}_{12} \\
\mathbf{X}_{13} \\
\mathbf{X}_{14} \\
\mathbf{X}_{15} \\
\mathbf{X}_{16} \\
\mathbf{X}_{17}\end{array}$ & $\begin{array}{r}\text { : Seed in } \\
\text { : Fruit ro } \\
: \alpha \text {-amyl } \\
\text { Total solub } \\
\text { : Capsaic } \\
\text { : Oleores } \\
\text { : ASTA }\end{array}$ & $\begin{array}{l}\text { ection }(\% \\
\text { disease } \\
\text { se activi } \\
\text { e seed pr } \\
\text { in }\left(\mu \mathrm{g} \mathrm{g}^{-1}\right. \\
\text { in }(\%) \\
\text { olor valu }\end{array}$ & $\begin{array}{l}\text { ) } \\
\text { ncidence } \\
\text { y ( } \mu \mathrm{g} \mathrm{ml} \\
\text { tein cont }\end{array}$ & $\begin{array}{l}\text { n ripe fruits }(\%) \\
\left.\min ^{-1} .\right) \\
\text { nt }\left(\mu \mathrm{g} \mathrm{g}^{-1}\right)\end{array}$ & \\
\hline
\end{tabular}


Table.2 Estimation of phenotypic correlation coefficients between seed quality attributes and germination

\begin{tabular}{|c|c|c|c|c|c|c|c|c|c|c|c|c|c|c|c|c|c|}
\hline & $\mathbf{X}_{1}$ & $\mathbf{X}_{2}$ & $\mathbf{X}_{3}$ & $\mathbf{X}_{4}$ & $X_{6}$ & $\mathbf{X}_{7}$ & $\mathbf{X}_{8}$ & $X_{9}$ & $\mathbf{X}_{10}$ & $X_{11}$ & $X_{12}$ & $\mathbf{X}_{13}$ & $X_{14}$ & $\mathbf{X}_{15}$ & $\mathbf{X}_{16}$ & $X_{17}$ & $\mathbf{X}_{5}$ \\
\hline$X_{1}$ & 1 & $0.6053^{* *}$ & $-0.3062 * *$ & $0.5047 * *$ & $-0.3291 * *$ & $0.6084 * *$ & $0.532 * *$ & $0.5887 * *$ & $0.4577 * *$ & $0.5743 * *$ & $0.4477 * *$ & $0.4876^{* *}$ & $0.4061^{* *}$ & $-0.5051 * *$ & $0.1868 * *$ & $0.5139 * *$ & $0.2816^{* *}$ \\
\hline $\mathbf{X}_{2}$ & & 1 & $-0.3445 * *$ & $0.3995 * *$ & $-0.2952 * *$ & $0.4838 * *$ & $0.4475 * *$ & $0.5547 * *$ & $0.4772 * *$ & $0.3692 * *$ & $0.3435^{* *}$ & $0.3764 * *$ & $0.2829 * *$ & $-0.3989 * *$ & 0.1383 & $0.2495 * *$ & $0.2997 * *$ \\
\hline $\mathbf{X}_{3}$ & & & 1 & $-0.5398^{* *}$ & $0.8228 * *$ & $-0.6637^{* *}$ & $-0.7374 * *$ & $-0.5517 * *$ & $-0.7284 * *$ & $-0.537 * *$ & $-0.4623 * *$ & $-0.5984 * *$ & $-0.4037 * *$ & $0.678^{* *}$ & -0.0686 & $-0.336^{* *}$ & $-0.8125^{* *}$ \\
\hline $\mathbf{X}_{4}$ & & & & 1 & $-0.627^{* *}$ & $0.6094 * *$ & $0.6764^{* *}$ & $0.6517^{* *}$ & $0.7158^{* *}$ & $0.6073^{* *}$ & $0.5414^{* *}$ & $0.9876^{* *}$ & $0.7862^{* *}$ & $-0.7846 * *$ & $0.2855^{* *}$ & $0.762 * *$ & $0.6566^{* *}$ \\
\hline$X_{6}$ & & & & & 1 & $-0.7278^{* *}$ & $-0.81 * *$ & $-0.5762 * *$ & $-0.7905 * *$ & $-0.5763 * *$ & $-0.4733 * *$ & $-0.6519 * *$ & $-0.4447 * *$ & $0.7141^{* *}$ & $-0.2492 * *$ & $-0.4027 * *$ & $-0.889 * *$ \\
\hline $\mathbf{X}_{7}$ & & & & & & 1 & $0.9682 * *$ & $0.8302^{* *}$ & $0.8227^{* *}$ & $0.6161^{* *}$ & $0.4852 * *$ & $0.6241^{* *}$ & $0.6225^{* *}$ & $-0.7477 * *$ & $0.245^{* *}$ & $0.5025^{* *}$ & $0.7296^{* *}$ \\
\hline $\mathbf{X}_{8}$ & & & & & & & 1 & $0.8212^{* *}$ & $0.9106^{* *}$ & $0.6211^{* *}$ & $0.4953^{* *}$ & $0.6966^{* *}$ & $0.684^{* *}$ & $-0.7993^{* *}$ & $0.2824^{* *}$ & $0.5484^{* * *}$ & $0.8694^{* *}$ \\
\hline$X_{9}$ & & & & & & & & 1 & $0.8757 * *$ & $0.552 * *$ & $0.455^{* *}$ & $0.665^{* *}$ & $0.6581 * *$ & $-0.6961 * *$ & $0.1717 * *$ & $0.4966 * *$ & $0.6276^{* * *}$ \\
\hline$X_{10}$ & & & & & & & & & 1 & $0.5901 * *$ & $0.4927^{* *}$ & $0.7373 * *$ & $0.7078 * *$ & $-0.7832 * *$ & $0.2715 * *$ & $0.5541 * *$ & $0.8991 * *$ \\
\hline$X_{11}$ & & & & & & & & & & 1 & $0.8712^{* *}$ & $0.6106^{* * *}$ & $0.4627 * *$ & $-0.7655^{* *}$ & $0.2012 * *$ & $0.5425^{* *} *$ & $0.5309 * *$ \\
\hline$X_{12}$ & & & & & & & & & & & 1 & $0.5462 * *$ & $0.423^{* *}$ & $-0.6076^{* *}$ & $0.2813^{* *}$ & $0.4788 * *$ & $0.4458^{* * *}$ \\
\hline$X_{13}$ & & & & & & & & & & & & 1 & $0.7786^{* * *}$ & $-0.7982^{* *}$ & $0.2585^{* * *}$ & $0.7454 * *$ & $0.6895^{* *}$ \\
\hline$X_{14}$ & & & & & & & & & & & & & 1 & $-0.6283^{* *}$ & 0.3724 & $0.6853^{* *}$ & $0.6082^{* *}$ \\
\hline$X_{16}$ & & & & & & & & & & & & & & & 1 & $0.5346^{* *}$ & $0.2786^{* *}$ \\
\hline$X_{17}$ & & & & & & & & & & & & & & & & 1 & $0.4885^{* *}$ \\
\hline
\end{tabular}

\section{$\mathrm{R}^{2}=0.9859 \quad$ Residual effect $=0.1186$}

Where,

$\mathbf{X}_{1} \quad:$ No. of seed fruit ${ }^{-1}$

$\mathbf{X}_{\mathbf{2}} \quad$ : Test weight $(\mathrm{g})$

$\mathbf{X}_{3} \quad$ : Electrical conductivity $\left(\mathrm{mS} \mathrm{cm}^{-1} \mathrm{~g}^{-1}\right)$

$\mathbf{X}_{\mathbf{4}} \quad$ : Total dehydrogenase activity $\left(\mathrm{A}^{0} 480 \mathrm{~nm}\right)$

$\mathbf{X}_{5} \quad$ : Germination (\%)

$\mathbf{X}_{\mathbf{6}} \quad$ : Fresh ungerminated seeds (\%)

$\mathbf{X}_{7} \quad$ : Seedling length $(\mathrm{cm})$

$\mathbf{X}_{\mathbf{8}} \quad$ : Seedling vigour index-I

$\mathbf{X}_{\mathbf{9}} \quad$ : Seedling dry weight $(\mathrm{mg})$

$\mathbf{X}_{10} \quad$ : Seedling vigour index-II
$\mathbf{X}_{11} \quad$ : Seed infection (\%)

$\mathbf{X}_{12} \quad$ : Fruit rot disease incidence on ripe fruits $(\%)$

$\mathbf{X}_{13} \quad: \alpha$-amylase activity $\left(\mu \mathrm{g} \mathrm{ml}^{1} \min ^{1}\right)$

$\mathbf{X}_{14} \quad$ : Total soluble seed protein content $\left(\mu \mathrm{g} \mathrm{g}^{-1}\right)$

$\mathbf{X}_{15} \quad$ : Capsaicin $\left(\mu \mathrm{g} \mathrm{g}^{-1}\right)$

$\mathbf{X}_{16} \quad$ : Oleoresin (\%)

$\mathbf{X}_{17}$ : ASTA color value 
Table.3 Estimation of phenotypic path coefficients between seed quality attributes and germination

\begin{tabular}{|c|c|c|c|c|c|c|c|c|c|c|c|c|c|c|c|c|c|}
\hline & $\mathbf{X}_{1}$ & $\mathbf{X}_{2}$ & $\mathbf{X}_{3}$ & $\mathbf{X}_{4}$ & $X_{6}$ & $\mathbf{X}_{7}$ & $\mathbf{X}_{8}$ & $X_{9}$ & $\mathbf{X}_{10}$ & $\mathbf{X}_{11}$ & $X_{12}$ & $\mathbf{X}_{13}$ & $X_{14}$ & $\mathbf{X}_{15}$ & $X_{16}$ & $X_{17}$ & $X_{5}$ \\
\hline $\mathbf{X}_{1}$ & 0.0038 & 0.0023 & -0.0012 & 0.0019 & -0.0012 & 0.0023 & 0.002 & 0.0022 & 0.0017 & 0.0022 & 0.0017 & 0.0018 & 0.0015 & -0.0019 & 0.0007 & 0.0019 & 0.2816 \\
\hline $\mathbf{X}_{2}$ & 0.0034 & 0.0056 & -0.0019 & 0.0022 & -0.0016 & 0.0027 & 0.0025 & 0.0031 & 0.0027 & 0.0021 & 0.0019 & 0.0021 & 0.0016 & -0.0022 & 0.0008 & 0.0014 & 0.2997 \\
\hline $\mathbf{X}_{3}$ & 0.0237 & 0.0266 & -0.0773 & 0.0417 & -0.0636 & 0.0513 & 0.057 & 0.0427 & 0.0563 & 0.0415 & 0.0357 & 0.0463 & 0.0312 & -0.0524 & 0.0053 & 0.026 & -0.8125 \\
\hline$X_{4}$ & -0.0579 & -0.0458 & 0.0619 & -0.1147 & 0.0719 & -0.0699 & -0.0776 & -0.0748 & -0.0821 & -0.0697 & -0.0621 & -0.1133 & -0.0902 & 0.09 & -0.0328 & -0.0874 & 0.6566 \\
\hline$X_{5}$ & 0.0747 & 0.067 & -0.1866 & 0.1422 & -0.2268 & 0.1651 & 0.1837 & 0.1307 & 0.1793 & 0.1307 & 0.1074 & 0.1479 & 0.1009 & -0.162 & 0.0565 & 0.0913 & -0.889 \\
\hline$X_{6}$ & -0.7929 & -0.6306 & 0.865 & -0.7943 & 0.9487 & -1.3034 & -1.2619 & -1.0822 & -1.0723 & -0.8031 & -0.6325 & -0.8135 & -0.8114 & 0.9745 & -0.3193 & -0.655 & 0.7296 \\
\hline $\mathbf{X}_{7}$ & 0.9793 & 0.8237 & -1.3574 & 1.2451 & -1.491 & 1.7822 & 1.8408 & 1.5117 & 1.6762 & 1.1432 & 0.9118 & 1.2822 & 1.2592 & -1.4714 & 0.5198 & 1.0095 & 0.8694 \\
\hline $\mathbf{X}_{8}$ & -0.0696 & -0.0656 & 0.0652 & -0.077 & 0.0681 & -0.0981 & -0.097 & -0.1182 & -0.1035 & -0.0652 & -0.0538 & -0.0786 & -0.0778 & 0.0823 & -0.0203 & -0.0587 & 0.6276 \\
\hline$X_{9}$ & 0.0838 & 0.0874 & -0.1334 & 0.1311 & -0.1447 & 0.1506 & 0.1667 & 0.1604 & 0.1831 & 0.1081 & 0.0902 & 0.135 & 0.1296 & -0.1434 & 0.0497 & 0.1015 & 0.8991 \\
\hline$X_{10}$ & -0.032 & -0.0206 & 0.0299 & -0.0338 & 0.0321 & -0.0343 & -0.0346 & -0.0307 & -0.0329 & -0.0557 & -0.0485 & -0.034 & -0.0258 & 0.0426 & -0.0112 & -0.0302 & 0.53 \\
\hline$X_{11}$ & 0.0192 & 0.0147 & -0.0198 & 0.0232 & -0.0203 & 0.0208 & 0.0212 & 0.0195 & 0.0211 & 0.0374 & 0.0429 & 0.0234 & 0.0181 & -0.0261 & 0.0121 & 0.0205 & 0.4458 \\
\hline$X_{13}$ & 0.0354 & 0.0273 & -0.0434 & 0.0716 & -0.0473 & 0.0453 & 0.0505 & 0.0482 & 0.0535 & 0.0443 & 0.0396 & 0.0725 & 0.0565 & -0.0579 & 0.0187 & 0.054 & 0.6895 \\
\hline$X_{14}$ & 0.0019 & 0.0013 & -0.0019 & 0.0036 & -0.002 & 0.0029 & 0.0031 & 0.003 & 0.0033 & 0.0021 & 0.0019 & 0.0036 & 0.0046 & -0.0029 & 0.0017 & 0.0031 & 0.6082 \\
\hline$X_{15}$ & 0.0079 & 0.0062 & -0.0106 & 0.0122 & -0.0111 & 0.0116 & 0.0125 & 0.0108 & 0.0122 & 0.0119 & 0.0095 & 0.0124 & 0.0098 & -0.0156 & 0.0023 & 0.0097 & -0.7482 \\
\hline$X_{16}$ & -0.0015 & -0.0011 & 0.0006 & -0.0024 & 0.0021 & -0.002 & -0.0023 & -0.0014 & -0.0022 & -0.0017 & -0.0023 & -0.0021 & -0.0031 & 0.0012 & -0.0083 & -0.0044 & 0.2786 \\
\hline$X_{17}$ & 0.0026 & 0.0013 & -0.0017 & 0.0039 & -0.0021 & 0.0026 & 0.0028 & 0.0025 & 0.0028 & 0.0028 & 0.0024 & 0.0038 & 0.0035 & -0.0032 & 0.0027 & 0.0051 & 0.4885 \\
\hline
\end{tabular}

$\mathrm{R}^{2}=0.9859 \quad$ Residual effect $=0.1186$

Where,

$\begin{array}{ll}\mathbf{X}_{\mathbf{1}} & : \text { No. of seed fruit }{ }^{-1} \\ \mathbf{X}_{\mathbf{2}} & \text { : Test weight }(\mathrm{g}) \\ \mathbf{X}_{\mathbf{3}} & \text { : Electrical conductivity }\left(\mathrm{mS} \mathrm{cm} \mathrm{cg}^{-1}\right) \\ \mathbf{X}_{\mathbf{4}} & \text { : Total dehydrogenase activity }\left(\mathrm{A}^{0} 480 \mathrm{~nm}\right) \\ \mathbf{X}_{\mathbf{5}} & : \text { Germination }(\%) \\ \mathbf{X}_{\mathbf{6}} & \text { : Fresh ungerminated seeds }(\%) \\ \mathbf{X}_{\mathbf{7}} & \text { : Seedling length }(\mathrm{cm}) \\ \mathbf{X}_{\mathbf{8}} & \text { : Seedling vigour index-I } \\ \mathbf{X}_{\mathbf{9}} & \text { : Seedling dry weight }(\mathrm{mg}) \\ \mathbf{X}_{\mathbf{1 0}} & : \text { Seedling vigour index-II }\end{array}$

$\mathbf{X}_{11} \quad$ : Seed infection $(\%)$

$\mathbf{X}_{12} \quad$ : Fruit rot disease incidence on ripe fruits (\%)

$\mathbf{X}_{13} \quad: \alpha$-amylase activity $\left(\mu \mathrm{g} \mathrm{ml}^{-1} \mathrm{~min}^{-1}\right)$

$\mathbf{X}_{14}$

$\mathbf{X}_{15}$

$\mathbf{X}_{16}$

$\mathbf{X}_{17}$

: Total soluble seed protein content $\left(\mu \mathrm{g} \mathrm{g}^{-1}\right)$

: Capsaicin $\left(\mu \mathrm{g} \mathrm{g}^{-1}\right)$

: Oleoresin (\%)

: ASTA color value 
Table.4 Estimation of phenotypic correlation coefficients between seed quality attributes and seed infection

\begin{tabular}{|c|c|c|c|c|c|c|c|c|c|c|c|c|c|c|c|c|c|}
\hline & $\mathbf{X}_{1}$ & $\mathbf{X}_{2}$ & $\mathbf{X}_{3}$ & $\mathbf{X}_{4}$ & $\mathbf{X}_{5}$ & $\mathbf{X}_{6}$ & $\mathbf{X}_{7}$ & $\mathbf{X}_{8}$ & $\mathrm{X}_{9}$ & $\mathbf{X}_{10}$ & $\mathbf{X}_{12}$ & $\mathbf{X}_{13}$ & $\mathrm{X}_{14}$ & $\mathbf{X}_{15}$ & $\mathrm{X}_{16}$ & $\mathbf{X}_{17}$ & $\mathrm{X}_{11}$ \\
\hline$X_{1}$ & 1 & $0.6053^{* *}$ & $-0.3062 * *$ & $0.5047^{* *}$ & $0.2816^{* * *}$ & $-0.3291 * *$ & $0.6084 * *$ & $0.532 * *$ & $0.5887 * *$ & $0.4577^{* *}$ & $0.4477^{* *}$ & $0.4876^{* *}$ & $0.4061 * *$ & $-0.5051 * *$ & $0.1868^{* *}$ & $0.5139 * *$ & $0.5743 * *$ \\
\hline $\mathbf{X}_{2}$ & & 1 & $-0.3445^{* *}$ & $0.3995^{* *}$ & $0.2997 * *$ & $-0.2952^{* *}$ & $0.4838^{* *}$ & $0.4475^{* *}$ & $0.5547 * *$ & $0.4772 * *$ & $0.3435^{* *}$ & $0.3764 * *$ & $0.2829 * *$ & $-0.3989 * *$ & 0.1383 & $0.2495^{* *}$ & $0.3692 * *$ \\
\hline $\mathbf{X}_{3}$ & & & 1 & $-0.5398 * *$ & $-0.8125^{* *}$ & $0.8228 * *$ & $-0.6637 * *$ & $-0.7374 * *$ & $-0.5517 * *$ & $-0.7284 * *$ & $-0.4623 * *$ & $-0.5984 * *$ & $-0.4037 * *$ & $0.678 * *$ & -0.0686 & $-0.336^{* *}$ & $-0.537 * *$ \\
\hline $\mathbf{X}_{4}$ & & & & 1 & $0.6566^{* *}$ & $-0.627 * *$ & $0.6094 * *$ & $0.6764 * *$ & $0.6517 * *$ & $0.7158^{* *}$ & $0.5414 * *$ & $0.9876^{* *}$ & $0.7862 * *$ & $-0.7846^{* *}$ & $0.2855^{* *}$ & $0.762 * *$ & $0.6073 * *$ \\
\hline$X_{5}$ & & & & & 1 & $-0.889 * *$ & $0.7296 * *$ & $0.8694 * *$ & $0.6276^{* *}$ & $0.8991 * *$ & $0.4458 * *$ & $0.6895 * *$ & $0.6082 * *$ & $-0.7482 * *$ & $0.2786^{* *}$ & $0.4885^{* *}$ & $0.5309 * *$ \\
\hline $\mathbf{X}_{6}$ & & & & & & 1 & $-0.7278^{* *}$ & $-0.81^{* *}$ & $-0.5762 * *$ & $-0.7905^{* *}$ & $-0.4733 * *$ & $-0.6519 * *$ & $-0.4447 * *$ & $0.7141^{* *}$ & $-0.2492^{* *}$ & $-0.4027^{* *}$ & $-0.5763 * *$ \\
\hline$X_{7}$ & & & & & & & 1 & $0.9682 * *$ & $0.8302 * *$ & $0.8227 * *$ & $0.4852 * *$ & $0.6241 * *$ & $0.6225^{* *}$ & $-0.7477 * *$ & $0.245^{* *}$ & $0.5025^{* *}$ & $0.6161^{* *}$ \\
\hline $\mathbf{X}_{8}$ & & & & & & & & 1 & $0.8212 * *$ & $0.9106^{* *}$ & $0.4953 * *$ & $0.6966^{* *}$ & $0.684^{* *}$ & $-0.7993 * *$ & $0.2824 * *$ & $0.5484 * *$ & $0.6211^{* *}$ \\
\hline$X_{9}$ & & & & & & & & & 1 & $0.8757^{* *}$ & $0.455^{* *}$ & $0.665^{* *}$ & $0.6581^{* *}$ & $-0.6961 * *$ & $0.1717^{* *}$ & $0.4966^{* *}$ & $0.552 * *$ \\
\hline $\mathbf{X}_{10}$ & & & & & & & & & & 1 & $0.4927 * *$ & $0.7373 * *$ & $0.7078^{* *}$ & $-0.7832 * *$ & $0.2715^{* *}$ & $0.5541^{* *}$ & $0.5901 * *$ \\
\hline$X_{12}$ & & & & & & & & & & & 1 & $0.5462 * *$ & $0.423^{* *}$ & $-0.6076 * *$ & $0.2813^{* *}$ & $0.4788^{* *}$ & $0.8712 * *$ \\
\hline $\mathbf{X}_{13}$ & & & & & & & & & & & & 1 & $0.7786^{* *}$ & $-0.7982 * *$ & $0.2585^{* *}$ & $0.7454 * *$ & $0.6106^{* *}$ \\
\hline$X_{14}$ & & & & & & & & & & & & & 1 & $-0.6283^{* *}$ & $0.3724 * *$ & $0.6853^{* *}$ & $0.4627^{* *}$ \\
\hline$X_{15}$ & & & & & & & & & & & & & & 1 & -0.1467 & $-0.6213^{* *}$ & $-0.7655^{* *}$ \\
\hline $\mathbf{X}_{16}$ & & & & & & & & & & & & & & & 1 & $0.5346^{* *}$ & $0.2012 * *$ \\
\hline$X_{17}$ & & & & & & & & & & & & & & & & 1 & $0.5425 * *$ \\
\hline
\end{tabular}

Where,

$\mathbf{X}_{1} \quad$ : No. of seed fruit ${ }^{-1}$

$\mathbf{X}_{2} \quad$ : Test weight (g)

$\mathbf{X}_{3} \quad$ : Electrical conductivity $\left(\mathrm{mS} \mathrm{cm}^{-1} \mathrm{~g}^{-1}\right)$

$\mathbf{X}_{\mathbf{4}} \quad$ : Total dehydrogenase activity $\left(\mathrm{A}^{0} 480 \mathrm{~nm}\right)$

$\mathbf{X}_{\mathbf{5}}$ : Germination (\%)

$\mathbf{X}_{\mathbf{6}}$ : Fresh ungerminated seeds (\%)

$\mathbf{X}_{7} \quad$ : Seedling length $(\mathrm{cm})$

$\mathbf{X}_{\mathbf{8}} \quad$ : Seedling vigour index-I

$\mathbf{X}_{\mathbf{9}} \quad$ : Seedling dry weight $(\mathrm{mg})$

$\mathbf{X}_{10}$ : Seedling vigour index-II
$\mathbf{X}_{11} \quad$ : Seed infection (\%)

$\mathbf{X}_{12}$ : Fruit rot disease incidence on ripe fruits (\%)

$\mathbf{X}_{13}: \alpha$-amylase activity $\left(\mu \mathrm{g} \mathrm{m} \mathrm{m}^{-1} \mathrm{~min}^{-1}\right)$

$\mathbf{X}_{14}$ : Total soluble seed protein content $\left(\mu \mathrm{g} \mathrm{g}^{-1}\right)$

$\mathbf{X}_{15}$ : Capsaicin $\left(\mu \mathrm{g} \mathrm{g}^{-1}\right)$

$\mathbf{X}_{16}$ : Oleoresin (\%)

$\mathbf{X}_{17}$ : ASTA color value 
Fig.1 Estimation of phenotypic path coefficients between seed quality attributes and germination

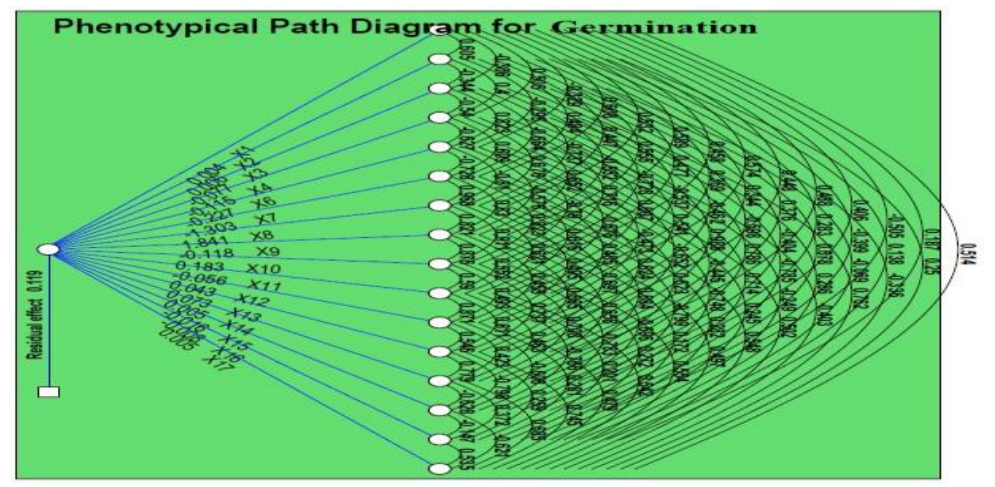

Where:

$\begin{array}{ll}\mathbf{X}_{1} & \text { : No. of seed fruit } \\ \mathbf{X}_{\mathbf{2}} & \text { : Test weight }(\mathrm{g}) \\ \mathbf{X}_{\mathbf{3}} & \text { : Electrical conductivity }\left(\mathrm{mS} \mathrm{cm}^{-1} \mathrm{~g}^{-1}\right) \\ \mathbf{X}_{\mathbf{4}} & \text { : Total dehydrogenase activity }\left(\mathrm{A}^{0} 480 \mathrm{~nm}\right) \\ \mathbf{X}_{\mathbf{5}} & \text { : Germination }(\%) \\ \mathbf{X}_{\mathbf{6}} & \text { : Fresh ungerminated seeds }(\%) \\ \mathbf{X}_{7} & \text { : Seedling length }(\mathrm{cm}) \\ \mathbf{X}_{\mathbf{8}} & \text { : Seedling vigour index-I } \\ \mathbf{X}_{9} & \text { : Seedling dry weight }(\mathrm{mg}) \\ \mathbf{X}_{10} & \text { : Seedling vigour index-II }\end{array}$

$\mathbf{X}_{11} \quad$ : Seed infection (\%)

$\mathbf{X}_{\mathbf{1 2}}$ : Fruit rot disease incidence on ripe fruits (\%)

$\mathbf{X}_{13} \quad: \alpha$-amylase activity $\left(\mu \mathrm{g} \mathrm{ml}^{-1} \mathrm{~min}^{-1}\right)$

$\mathbf{X}_{14}$ :Total soluble seed protein content $\left(\mu \mathrm{g} \mathrm{g}^{-1}\right)$

$\mathbf{X}_{15} \quad$ : Capsaicin $\left(\mu \mathrm{g} \mathrm{g}^{-1}\right)$

$\mathbf{X}_{\mathbf{1 6}}$ : Oleoresin (\%)

$\mathbf{X}_{17}$ : ASTA color value

Fig.2 Estimation of phenotypic path coefficients between seed quality attributes and seed infection

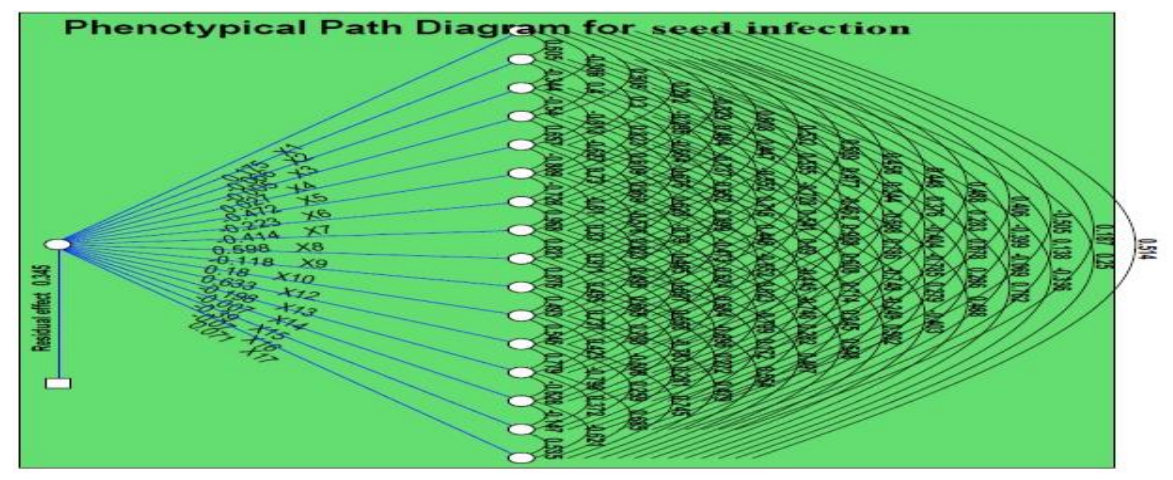

Where:

$\mathbf{X}_{1} \quad$ : No. of seed fruit ${ }^{-1}$

$\mathbf{X}_{\mathbf{2}} \quad$ : Test weight $(\mathrm{g})$

$\mathbf{X}_{\mathbf{3}}$ : Electrical conductivity $\left(\mathrm{mS} \mathrm{cm}^{-1} \mathrm{~g}^{-1}\right)$

$\mathbf{X}_{\mathbf{4}} \quad$ : Total dehydrogenase activity $\left(\mathrm{A}^{0} 480 \mathrm{~nm}\right)$

$\mathbf{X}_{\mathbf{5}} \quad$ : Germination (\%)

$\mathbf{X}_{\mathbf{6}} \quad$ : Fresh ungerminated seeds $(\%)$

$\mathbf{X}_{7} \quad$ : Seedling length $(\mathrm{cm})$

$\mathbf{X}_{\mathbf{8}} \quad$ : Seedling vigour index-I

$\mathbf{X}_{\mathbf{9}} \quad$ : Seedling dry weight $(\mathrm{mg})$

$\mathbf{X}_{10} \quad$ : Seedling vigour index-II
$\mathbf{X}_{11} \quad$ : Seed infection (\%)

$\mathbf{X}_{12}$ : Fruit rot disease incidence on ripe fruits (\%)

$\mathbf{X}_{13} \quad: \alpha$-amylase activity $\left(\mu \mathrm{g} \mathrm{ml}^{-1} \mathrm{~min}^{-1}\right)$

$\mathbf{X}_{14}$ : Total soluble seed protein content $\left(\mu \mathrm{g} \mathrm{g}^{-1}\right)$

$\mathbf{X}_{15}$ : Capsaicin $\left(\mu \mathrm{g} \mathrm{g}^{-1}\right)$

$\mathbf{X}_{16} \quad$ : Oleoresin (\%)

$\mathbf{X}_{\mathbf{1 7}}$ : ASTA color value 


\section{Association of characters}

\section{Germination (\%)}

The characters such as number of seed fruit ${ }^{-1}$ (0.2816), 1000 seed weight (0.2997), total dehydrogenase activity (0.6566), seedling length (0.7296), vigour index I (0.8694), vigour index II (0.8991) seed infection (0.5309), fruit rot disease incidence on ripe fruits (0.4458), $\alpha$-amylase activity (0.6895), total soluble seed protein content $(0.6082)$, oleoresin (0.2786), ASTA color value (0.4885) and seedling dry weight (0.6276) showed significant positive correlation with germination per cent while electrical conductivity (-0.8125), fresh ungerminated seeds (-0.8890), capsaicin (-0.7482) showed significant negative correlation with germination per cent (Table 2.).

Number of seed fruit ${ }^{-1}, 1000$ seed weight, total dehydrogenase activity, seedling length, seedling vigour index-I, seedling vigour index-I, seed infection, fruit rot disease incidence on ripe fruits, $\alpha$-amylase activity, total soluble seed protein content, oleoresin, ASTA colour value and seedling dry weight manifested significant positive correlation with germination per cent suggested the effectiveness of selection based on these characters in improving the germination per cent while electrical conductivity, fresh ungerminated seeds and capsaicin showed significant negative correlation with germination per cent indicated limited success of selection for these traits in improving germination per cent.

\section{Seed infection $(\%)$}

The characters such as number of seed fruit ${ }^{-1}$ (0.5743), 1000 seed weight $(0.3692)$, total dehydrogenase activity (0.6073), germination (0.5309), seedling length (0.6161), vigour index I (0.6211), seedling dry weight
(0.5520), vigour index II (0.5901), fruit rot disease incidence on ripe fruits (0.8712), $\alpha$ amylase activity (0.6106), Total soluble seed protein content (0.4627), oleoresin (0.2012), ASTA color value (0.5425) showed significant positive correlation with seed infection while Electrical conductivity ($0.5370)$, fresh ungerminated seeds (-0.5763), capsaicin (-0.7655) showed significant negative correlation with seed infection (Table 3) .

The characters such as number of seed fruit ${ }^{-1}$, 1000 seed weight, total dehydrogenase activity, germination, seedling length, seedling vigour index-I, seedling dry weight, seedling vigour index-II, fruit rot disease incidence on ripe fruits, $\alpha$-amylase activity, total soluble seed protein content, oleoresin and ASTA colour value showed significant positive correlation with seed infection suggested the effectiveness of selection based on these characters in improving the seed infection. While, electrical conductivity, fresh ungerminated seeds and capsaicin showed significant negative correlation with seed infection indicated limited success of selection for these traits in improving seed infection.

\section{Direct effect of different characters on seed germination per cent at phenotypic level}

At the phenotypic level, seedling vigour index-I recorded the highest positive direct effect on germination per cent, followed by seedling vigour index-II which was of considerable magnitude. number of seed fruit $^{-1}, 1000$ seed weight, fruit rot disease incidence on ripe fruits, $\alpha$-amylase activity, total soluble seed protein content and ASTA colour value recorded positive direct effects on germination per cent but their magnitude were low. However, seedling length recorded the highest negative direct effect on germination per cent followed by fresh 
ungerminated seeds, seedling dry weight, and total dehydrogenase activity. Electrical conductivity, seed infection, capsaicin and oleoresin recorded negative direct effect on germination per cent but their magnitude were low (Fig. 1).

\section{Direct effect of different characters on seed infection at phenotypic level}

At the phenotypic level, fruit rot disease incidence on ripe fruits recorded the highest positive direct effect on seed infection, followed by vigour index I which was of considerable magnitude. Number of seed fruit $^{-1}$, total dehydrogenase activity, vigour index ii and ASTA colour value recorded positive direct effects on seed infection but their magnitude were low. Whereas, germination per cent recorded the highest positive direct effect on seed infection followed by seedling length, capsaicin which was of considerable magnitude. 1000 seed weight, electrical conductivity, fresh ungerminated seeds, seedling dry weight, $\alpha$ amylase activity, total soluble seed protein content and oleoresin recorded the negative direct effect on seed infection but their magnitude were low (Fig. 2). Such findings were also reported by Dewey and Lu, 1957 in wheat grass, Jayashree et al., 2007 and Sarkar et al., 2009 for path analysis of morphological characters in hot pepper.

\section{References}

Anon., 2003, Text book on the genus Capsicum, edited by Amit Kishna De, $2^{\text {nd }}$ edn, published by Taylor and Francis, Inc. New York and London, 71-86 pp.

Anon., 2006, Package of practices for horticultural crops, University of Agricultural Sciences, Bangalore5600 065. pp 184-189.

Arnon, B. C., Yelena, B., Matthew, F.,
Michael, M., Byoung, C.K., Ilan, P. and Molly, J., 2006, QTL analysis for capsaicinoid content in Capsicum. Theor. Appl. Genet. 113: 1481-1490.

Bosland, P.W. and Votava, E. J., 2000, Peppers: Vegetable and spice capsicums. Crop Production Science in Horticulture series 22, CAB International Publishing, Wallingford, England, UK. 84 pp.

Burton, G. W. and Dewane, E. M., 1953, Estimating heritability in tall Fesue (Fistula aundanaceae) from replicated clonal material. Agron. J., 45: 478-481.

Claudinei Andreoli, Manoel Carlos Bassoi, and Dionisio Brunetta, 2006, Genetic control of seed dormancy and preharvest sprouting in wheat. Sci. Agric. (Piracicaba, Braz.), 63(6): 564-566.

Cochran, M. G. and Cox, G. M., 1959, Experimental designs. Asian Printing House, New Delhi.

Dewey, D. R. and Lu, K. K., 1957, A correlation and path coefficient analysis of components of crested wheat grass seed production. Agron. J., 51: 515-520.

Ekbote, S. D., Padaganur, G. M., Patil, M. S., and Chattannavar, S. N., 1997, Studies on the cultural and nutritional aspects of Colletotrichum gloeosporoioides, the causal organism of mango anthracnose. J. Mycol. \& Pl. Pathol., 27: 229-230.

Gadal, M. C., Manjunath, A., Nehru, S. D. and Rudresh, N. S., 2003, Studies on association of fruit colour with other traits in chilli (Capsicum annuum L.). Indian Journal of Genetics and Plant Breeding. 63(2): 183-184.

Goulden, C, 1959, Methods of Statistical Analysis. Asia Publication House, Calcutta.

Hanson, G. H., Robinson, H. F., and 
Comstock, R. E., 1956, Biometrical studies in yield in segregating populations of Korean Laspedez. Agron, J. 48: 267-282.

Hedau, N. K., Saha, S., Singh, G., Gahlain, A., Mahajan, V., and Gupta, H. S., 2008, Analysis of genetic variability for nutritional quality traits in hot pepper (Capsicum annuum L.). $J$. Plant Genet. Resour. 21(2): 85-89.

Hegde, G. M., and Kulkarni, S., 2001, Vulnerable infection stage of chilli fruit by Colletotrichum capsici (Sydow) Butler and Bisby. Karnataka J. Agric. Sci., 14: 162-163.

ISTA, 1996, International Rules for Seed Testing. Seed Sci. and Technol. 23(2): 263-281.

Jayashree Wasule, Potdukhe, N.R., Deshmukh, D.T., and Neelam Dheva, 2007, Path analysis in chilli. Annals of Plant Physiology. 21(1): 130-131.

Johnson, H. W., Robinson, H. F. and Comstock, R. E., 1955, Genotypic and phenotypic correlations in Soybean and their application in selection, Agron. J., 47: 477-483.

Manju, P, R. and Sreelathakumary, I., 2002, Quality parameters in hot chilli (Capsicum chinense Jacq.). Journal of Tropical Agriculture. 40(1/2): 7-10.

Mini, S. and Khader, K. M. A., 2004, Variability, heritability and genetic advance in wax type chilli (Capsicum annuum L.). Capsicum and Eggplant Newsletter. 23: 49-52.

Mini, S. and Khader, K. M. A., 2004, Variability, heritability and genetic advance in wax type chilli (Capsicum annuum L.). Capsicum and Eggplant Newsletter. 23: 49-52.

Naresh, P., Reddy, K. M., Shivashankara, K. S. and George, C. M. 2013, Genotypic variation for biochemical compounds in capsicum. Indian J. of Horticulture. 70(1): 43-47.
Pitchumuthu and Pappiah, 1995, Heritability studies in chilli. J. Maharashtra Agric. Universities 20: 348-350.

Ranganna, 1996, Manual Analysis of Fruit and Vegetable Products, edn.2, Tata Mc Graw Hill Publishing Co. Ltd., New Delhi, India.

Ritesh, M., Dangi, R. S., Dass, S. C. and Malhotra, R. C., 2000, The hottest chilli variety in India. Current Sci., 79 (3): 287-288.

Robinson, H. F., Comstock, R. E., and Harvey, P. H., 1949, Estimates of heritability and degree of dominance in corn. Agron. J.41: 353-359.

Sadasivam, S. and Manickam, A., 1996, Biochemical methods, 2nd edn. Pub: New Age International (p) Limited, New Delhi, pp. 199-2001 RANGANNA, 1996, Manual Analysis of Fruit and Vegetable Products, edn.2, Tata Mc Graw Hill Publishing Co. Ltd., New Delhi, India.

Sarkar, S., Murmu, D., Chattopadhyay, A. and Hazra, P., 2009, Genetic variability, correlation and path analysis of some morphological characters in chilli. Journal of Crop and Weed. 5(1): 162-166.

Shiva, K. N., Gobinath, P., Zachariah, T. J. and Leela, N. K., 2014, Variability in quality attributes of paprika and paprika alike chillies (Capsicum annuum L.). J. Spices and Aromatic Crops. 23(1): 17-25.

Sundararaj, N., Nagaraj, S., Vekara Ramu, M. N. and Jagannath, M. K., 1972, Designs and analysis of field experiments. Uni. Agri. Sci., Hebbal, Bangalore. India.

Tewksbury, J. J. and Nabhan, G. P., 2001, Directed deterrence by capsaicin in chillies. Nature. 412: 403-404.

Verma, S. K., Singh, R. K. and Arya, R. R., 2004, Genetic variability and 
correlation studies in chillies. Progressive Horticulture. 36(1): 113117.

Yudhvir Singh and Madhu Sharma, 2008, Association of characters and their direct and indirect contribution for green chilli (Capsicum annuum L.) improvement. Haryana Journal of Horticultural Sciences. 37 (3/4): 345-
348.

Yudhvir Singh, Madhu Sharma and Akhilesh sharma, 2009, Genetic variation, association of characters, and their direct and indirect contributions for improvement in chilli peppers. International Journal of Vegetable Science. 15(4): 340-368.

\section{How to cite this article:}

Nagaraju, K. S., K. P. R. Prasanna, A. Mohan Rao and Aruna, K. 2020. Variability and Association of Capsaicin and Oleoresin on Seed Quality in Hot Pepper (Capsicum spp). Int.J.Curr.Microbiol.App.Sci. 9(11): 31-42. doi: https://doi.org/10.20546/ijcmas.2020.911.004 